\title{
Current-Induced Forces and Hot Spots in Biased Nanojunctions
}

\author{
Lu, Jing Tao; Christensen, Rasmus Bjerregaard; Wang, Jian-Sheng; Hedegård, Per; Brandbyge, Mads
}

Published in:

Physical Review Letters

Link to article, DOI:

10.1103/PhysRevLett.114.096801

Publication date:

2015

Document Version

Publisher's PDF, also known as Version of record

Link back to DTU Orbit

Citation (APA):

Lu, J. T., Christensen, R. B., Wang, J-S., Hedegård, P., \& Brandbyge, M. (2015). Current-Induced Forces and Hot Spots in Biased Nanojunctions. Physical Review Letters, 114(9), 096801.

https://doi.org/10.1103/PhysRevLett.114.096801

\section{General rights}

Copyright and moral rights for the publications made accessible in the public portal are retained by the authors and/or other copyright owners and it is a condition of accessing publications that users recognise and abide by the legal requirements associated with these rights.

- Users may download and print one copy of any publication from the public portal for the purpose of private study or research.

- You may not further distribute the material or use it for any profit-making activity or commercial gain

- You may freely distribute the URL identifying the publication in the public portal 


\title{
Current-Induced Forces and Hot Spots in Biased Nanojunctions
}

\author{
Jing-Tao Lü, ${ }^{1,2,3,{ }^{*}}$ Rasmus B. Christensen, ${ }^{2}$ Jian-Sheng Wang, ${ }^{4}$ Per Hedegård, ${ }^{3}$ and Mads Brandbyge ${ }^{5}$ \\ ${ }^{1}$ School of Physics and Wuhan National High Magnetic Field Center, Huazhong University of Science and Technology, \\ 430074 Wuhan, China \\ ${ }^{2}$ Department of Micro- and Nanotechnology, Technical University of Denmark, 2800 Kongens Lyngby, Denmark \\ ${ }^{3}$ Niels Bohr Institute, Nano-Science Center, University of Copenhagen, 2100 Copenhagen Ø, Denmark \\ ${ }^{4}$ Department of Physics, National University of Singapore, 117551 Singapore, Republic of Singapore \\ ${ }^{5}$ Center for Nanostructured Graphene $(\mathrm{CNG})$, Department of Micro- and Nanotechnology, \\ Technical University of Denmark, 2800 Kongens Lyngby, Denmark
}

(Received 28 September 2014; published 4 March 2015)

\begin{abstract}
We investigate theoretically the interplay of current-induced forces (CIFs), Joule heating, and heat transport inside a current-carrying nanoconductor. We find that the CIFs, due to the electron-phonon coherence, can control the spatial heat dissipation in the conductor. This yields a significant asymmetric concentration of excess heating (hot spot) even for a symmetric conductor. When coupled to the electrode phonons, CIFs drive different phonon heat flux into the two electrodes. First-principles calculations on realistic biased nanojunctions illustrate the importance of the effect.
\end{abstract}

DOI: 10.1103/PhysRevLett.114.096801

PACS numbers: 73.63.Rt, 85.65.+h

Introduction.-Current-induced forces (CIFs) and Joule heating both originate from the coupling between electrons and phonons [1], one of the most fundamental many-body interactions responsible for a wide range of phenomena in molecular and condensed-matter physics. Their vital role in maintaining the electronic device stability is further promoted at the nanoscale. Our understanding of the two closely related effects, especially their interplay in nanoand atomic conductors is still under development [2-10]. Several forces, present only in the nonequilibrium situation, have been discovered theoretically. Among them are the nonconservative (NC) "wind force" and the Berry-phase (BP) induced pseudomagnetic force. Different from stochastic Joule heating [11-23], the NC and BP forces can generate deterministic energy and momentum transfer between the current-carrying electrons and the vibrations in the conductor [2-6]. In carefully designed devices, this effect may be used to drive atomic motors [2,7]. Furthermore, it can also impact the stability of the device $[3,24,25]$. To this end, the vibrational or phononic [1] heat transport and heat distribution in the presence of current flow become an urgent problem to investigate.

The electrode phonons play an important role as heat sinks for the locally dissipated Joule heat in the conductor [15]. However, the effects on the heat transport of the deterministic CIFs and the momentum transfer from the current have so far not been explored. To address this question, we go beyond the previous treatments [3,26] considering localized vibrations in the conductor and include coupling to the phonons in the electrodes [27]. Employing the semiclassical generalized Langevin equation (SGLE) [26,28-30], we find that, in addition to energy transfer, the CIFs also influence how the excess vibrational energy is distributed in the junction and transported to the electrodes.
Using first-principles calculations, we demonstrate how symmetric current-carrying nanojunctions typically possess a significant asymmetric excess heat distribution with heat accumulation at hot spots in the junction. At the same time, the phonon heat flow to the two electrodes differs. This behavior is governed by the phases of the electron and phonon wave functions and is a result of electron-hole pair symmetry breaking in the electronic structure. It will have important implications and should be taken into account when considering junction disruption at high bias [24,31].

Method.-In the SGLE approach, we adopt the two-probe transport setup, where a "bottleneck" nanojunction (system) is connected to left $(L)$ and right $(R)$ electrodes. We consider the case where the system region is characterized by a significant current density and deviation from equilibrium. The current-carrying electrons are treated as a nonequilibrium bath, coupling linearly with the system displacement, while the remaining atoms in $L$ and $R$ form two phonon baths interacting with the system also via a linear coupling. The electron-phonon (e-ph) coupling Hamiltonian can be written as

$$
H_{\mathrm{e}-\mathrm{ph}}=\sum_{i, j, k} M_{i j}^{k}\left(c_{i}^{\dagger} c_{j}+\text { H.c. }\right) \hat{u}_{k} .
$$

Here, $\hat{u}_{k}=\sqrt{m_{k}} \hat{x}_{k}$ is the mass-normalized displacement away from the equilibrium position of the $k$ th atomic degrees of freedom, with $m_{k}$ the mass and $\hat{x}_{k}$ the displacement operator from equilibrium position; $c_{i}^{\dagger}\left(c_{j}\right)$ is the electron creation (annihilation) operator for the $i$ th $(j$ th) electronic state in the junction. The coupling matrix $M_{i j}^{k}$ is local in real space, nonzero in the system, and neglected in $L, R$. We treat the e-ph interaction perturbatively using the electron and phonon states obtained from the Born-Oppenheimer 
approximation. In order to focus on the effect of CIFs, we will ignore the change of Hamiltonian due to the applied voltage.

The SGLE describing the dynamics of the system atoms reads

$\ddot{U}(t)-F(U(t))=-\int{ }^{t} \Pi^{r}\left(t-t^{\prime}\right) U\left(t^{\prime}\right) d t^{\prime}+f(t)$,

where $U$ is a vector composed of the mass-normalized displacements of the system, and $F(U(t))$ is the force vector from the potential of the isolated system. We adopt the harmonic approximation $F(U(t))=-K U(t)$, with $K$ being the dynamical matrix. The effect of all bath degrees of freedom is hidden in the terms on the right-hand side of the SGLE. Each of them contains separate contributions from the $L, R$ phonons and the electron bath (e), such that $\Pi^{r}=\Pi_{L}^{r}+\Pi_{R}^{r}+\Pi_{e}^{r}$ and $f=f_{L}+f_{R}+f_{e}$. The phonon self-energy $\Pi^{r}$ describes the time-delayed backaction of the bath on the system due to its motion [3,26,28-30]. The second quantum term $f(t)$ is a random force (noise) due to the thermal or current-induced fluctuation of the bath variables. It is characterized by the correlation matrix $\left\langle f_{\alpha}(t) f_{\alpha}^{T}\left(t^{\prime}\right)\right\rangle=S_{\alpha}\left(t-t^{\prime}\right)$, with $\alpha=L, R, e$. The two phonon baths $(L$ and $R$ ) are assumed to be in thermal equilibrium. Their noise correlation $S_{L(R)}$ is related to $\Pi_{L(R)}^{r}$ through the fluctuation-dissipation theorem $S_{L(R)}(\omega)=\left[n_{B}(\omega, T)+\right.$ $\left.\frac{1}{2}\right] \Gamma_{L(R)}(\omega)$ with $\Gamma_{L(R)}(\omega)=-2 \operatorname{Im} \Pi_{L(R)}^{r}(\omega)$ and $n_{B}$ the Bose distribution function (using atomic units, $\hbar=1$ ). Because of the electrical current, the electronic bath is not in equilibrium. We define the coupling-weighted electron-hole pair density of states as $[3,26]$

$$
\begin{aligned}
\Lambda_{k l}^{\alpha \beta}(\omega)= & 2 \sum_{m, n}\left\langle\psi_{m}\left|M^{k}\right| \psi_{n}\right\rangle\left\langle\psi_{n}\left|M^{l}\right| \psi_{m}\right\rangle\left[n_{F}\left(\varepsilon_{n}-\mu_{\alpha}\right)\right. \\
& \left.-n_{F}\left(\varepsilon_{m}-\mu_{\beta}\right)\right] \delta\left(\varepsilon_{n}-\varepsilon_{m}-\omega\right),
\end{aligned}
$$

with $n_{F}$ the Fermi-Dirac distribution and $\psi_{n}$ the electron scattering state originating from the $n$th channel of electrode $\alpha$ when there is no e-ph interaction. The noise correlation and the backaction term of the electron bath can now be written as

$$
\begin{gathered}
S_{e}(\omega)=-2 \pi \sum_{\alpha \beta}\left[n_{B}\left[\omega-\left(\mu_{\alpha}-\mu_{\beta}\right)\right]+\frac{1}{2}\right] \Lambda^{\alpha \beta}(\omega), \\
\Pi_{e}^{r}(\omega)=-\frac{1}{2}\left[\mathcal{H}\left\{\Gamma_{e}\left(\omega^{\prime}\right)\right\}(\omega)+i \Gamma_{e}(\omega)\right], \\
\Gamma_{e}(\omega)=-2 \pi \sum_{\alpha \beta} \Lambda^{\alpha \beta}(\omega),
\end{gathered}
$$

where $\mathcal{H}\{A\}$ is the Hilbert transform of $A$.

In the absence of electrical current, the electrons serve as an equilibrium thermal bath, similar to phonons. However, in the presence of current, the term $\left(\sim \operatorname{Im} \Lambda_{k l}^{R L}, k \neq l\right)$ becomes important. It may coherently couple two vibrational modes $(\mathrm{kl})$ inside the system leading to nonzero $\mathrm{NC}$ and BP forces. In Eq. (3) we observe that these effects depend on the phase of the electronic wave function and, thus, the direction of electronic current. Furthermore, the coherent coupling breaks time-reversal symmetry of the noise correlation function $S_{e}\left(t-t^{\prime}\right) \neq S_{e}\left(t^{\prime}-t\right)$. Hereafter, we denote these forces by "asymmetric CIFs" and focus on their role for the excess heat distribution and heat transport in the junction.

We will consider the case where all baths are at the same temperature $(T)$ and the electron bath is subject to a nonzero voltage bias $\left(e V=\mu_{L}-\mu_{R}\right)$. To look at the excess heating, we calculate the kinetic energy of atom $n$ from its local displacement correlation function and obtain

$$
E_{n}=\sum_{\sigma=x, y, z} \int_{0}^{+\infty} \omega^{2} \operatorname{diag}\left\{D^{r} S D^{a}\right\}_{n, \sigma}(\omega) \frac{d \omega}{2 \pi} .
$$

Here $D^{r}\left(D^{a}\right)$ is the $e V$-dependent phonon retarded (advanced) Green's function, $S$ is the sum of noise correlation function from all the baths, and $\operatorname{diag}\{A\}_{n, \sigma}$ means the diagonal matrix element of $A$, corresponding to the $n$th atom's $\sigma$ degrees of freedom.

To study heat transport, we calculate the phonon heat current flowing into the bath $L$ as the product of the velocity of the system degrees of freedom and the force exerted on them by bath $L$. Applying time average, using the solution of the SGLE, we arrive at a Landauer-like expression (Sec. I, Supplemental Material [32])

$$
\begin{aligned}
J_{L}= & -\int_{-\infty}^{+\infty} \omega \operatorname{tr}\left[\Gamma_{L}(\omega) D^{r}(\omega) \Lambda^{R L}(\omega) D^{a}(\omega)\right] \\
& \times\left[n_{B}(\omega+e V)-n_{B}(\omega)\right] d \omega .
\end{aligned}
$$

Defining the time-reversed phonon spectral function from the left bath $\tilde{\mathcal{A}}_{L}=D^{a} \Gamma_{L} D^{r}$ and similarly $\mathcal{A}_{e}=D^{r} \Lambda^{R L} D^{a}$, we can write the trace in Eq. (8) in different forms

$$
\operatorname{tr}\left[\Gamma_{L} D^{r} \Lambda^{R L} D^{a}\right]=\operatorname{tr}\left[\Gamma_{L} \mathcal{A}_{e}\right]=\operatorname{tr}\left[\Lambda^{R L} \tilde{\mathcal{A}}_{L}\right] .
$$

Equation (8) is analogous to the Landauer or nonequilibrium Green's function formula for electron or phonon transport. In our present case, the energy current is driven by a nonthermal electron bath with the bias showing up in the Bose distributions and in the coupling function $\Lambda^{R L}$ between phonons and electrical current. The two forms in Eq. (9) emphasize two aspects of the problem. In the first version, emphasis is on the coupling $\Gamma_{L}$ of the system vibrations as described by $\mathcal{A}_{e}$ to the phonons of the leads. This is a general formula, which does not explicitly depend on the situation we are considering here, namely, that the source of energy is the nonequilibrium electron bath. This aspect is emphasized in the second version. Here, the 
coupling to the electrical current $\Lambda^{R L}$ is made explicit, and the complete phonon system including the coupling to leads is in the function $\tilde{\mathcal{A}}_{L}$. In both forms, the asymmetric CIFs show up in the different versions of the $\mathcal{A}$ functions. The forces are responsible for the buildup of vibrational energy inside the junction, a fact that is present in the two phonon Green's functions $D^{r}$ and $D^{a}$. Apart from this effect, the nonequilibrium nature of the electron system shows up in the explicit factor $\Lambda^{R L}$ in the second version of Eq. (9). This will develop an imaginary part that is not present in equilibrium.

Applying these formulas to a minimal model, in Sec. II of the Supplemental Material [32] we have shown analytically that the asymmetric CIFs, especially the NC force, generate an asymmetric phonon heat flow and energy distribution, even for a left-right symmetric system.

First-principles calculations.-Next, we turn to numerical calculation for two concrete nanojunctions. We use SIESTA and TRANSIESTA $[33,34]$ to calculate the electronic transport, vibrational modes, e-ph coupling employing Ref. [35], and coupling to electrode phonons using Ref. [36], with similar parameters. The effect of current on the stability of gold single-atomic junctions has been studied for more than a decade [31,37]. Here, we first consider a symmetric single-atom gold chain between two $\mathrm{Au}(100)$ electrodes $[38,39]$. The results are summarized in Figs. 1 and 2. The structure of the chain is shown in the inset of Fig. 2. We have previously [40] studied the asymmetric forces in this system neglecting the coupling to electrode phonons.

Figure 1 shows the average excess kinetic energy $\left[\Delta E_{n}=E_{n}(\mathrm{eV})-E_{n}(0)\right]$ [41-46] of atoms along the chain for three different Fermi levels $E_{F}$. The structure is almost mirror symmetric. When we turn off the asymmetric $\operatorname{CIF}\left(\operatorname{Im} \Lambda^{R L}=0\right)$ as in previous studies [14,47], the heating profile follows this symmetry. However, once we include them, the kinetic energy of one side becomes many times higher than that of the other. Meanwhile, the total kinetic energy stored in the system increase significantly. Further analysis shows that both effects are due to the NC force (Fig. 2 in the Supplemental Material [32]).

We now turn to the phonon heat current calculated using Eq. (8), shown in Fig. 2(a). The inclusion of the asymmetric CIF drives a much larger heat current into the $L$ bath. Intuitively, this is due to the asymmetric energy accumulation induced by the $\mathrm{NC}$ force, e.g., modifying $D^{r} / D^{a}$ in Eqs. (8) and (9). However, there is another contribution at low bias. Ignoring the bias-induced change of $\tilde{\mathcal{A}}_{L}$, we get opposite heat flow into $L$ and $R\left(J_{L}=-J_{R}\right)$ due to $\operatorname{tr}\left[\operatorname{Im} \Lambda^{R L} \operatorname{Im} \tilde{\mathcal{A}}_{L}^{0}\right]$. This term drives asymmetric heat flow even in the linear response regime, contributing with a correction to the thermoelectric Peltier coefficient (Sec. I (A) of the Supplemental Material [32]). In the next section, we will show that it can be understood as asymmetric excitation of left- and right-traveling phonon waves.

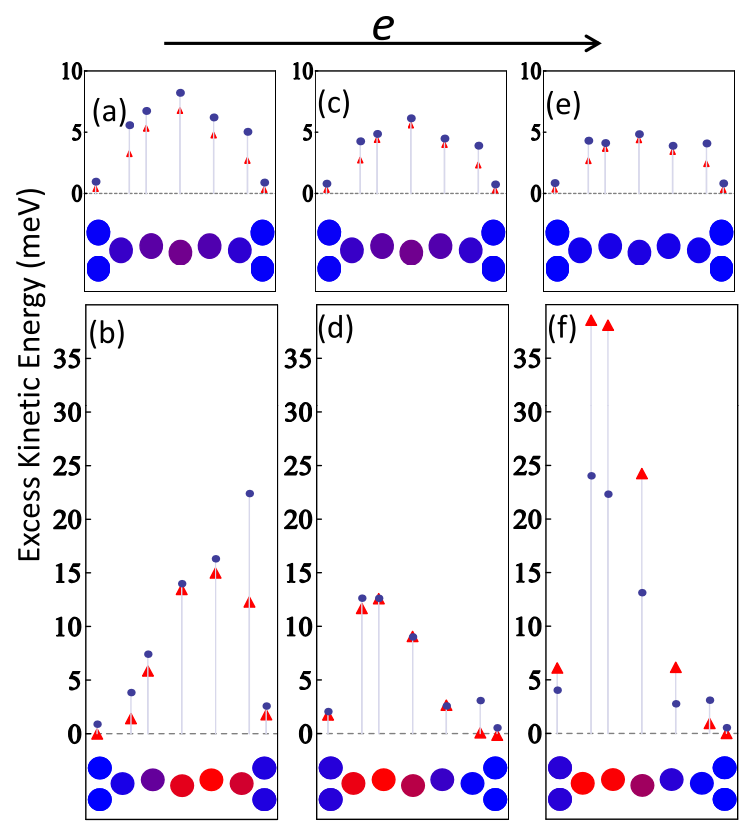

FIG. 1 (color online). Excess kinetic energy of each atom in a gold chain [inset of Fig. 2(a)] at $V=1.0 \mathrm{~V}, T=300 \mathrm{~K}$, with (bottom) and without (top) the asymmetric CIFs. The total energy difference between the two cases is due to the nonconservative force contribution. The blue dots and the colored plot of each atom are from the full calculation. The asymmetric heating is qualitatively reproduced by only considering electron coupling with vibrational modes (1) and (2) in the inset of Fig. 2(a), as shown by red triangles. [(a), (b)] $E_{F}=-0.3 \mathrm{eV},[(\mathrm{c}),(\mathrm{d})]$ $E_{F}=0$, and [(e), (f)] $E_{F}=0.2 \mathrm{eV}$. The arrow indicates the current direction.

From Figs. 1(b)-1(d) and 2(b), we see that the position of $E_{F}$ is controlling the direction and magnitude of the asymmetry. According to the analysis in Sec. IV of the Supplemental Material [32], this could be due to the phase change of the electronic wave function with $E_{F}$. Thus, we expect that the direction of electron flow is essential in the description of the atomic dynamics in the junction, as indicated in recent experiments [8].

The second system we consider is an armchair graphene nanoribbon with partial hydrogen passivation, shown in Fig. 3(a). This example is inspired by experiments showing current-induced edge reconstructions in graphene [48] where the physical mechanism was attributed to Joule heating [49]. In Fig. 3(a), the four pairs of unpassivated carbon dimers give rise to localized high-frequency vibrations interacting strongly with electrical current. Consequently, the excess energy is mainly stored in the dimers and nearby atoms [Figs. 3(b) and 3(d)], consistent with the experimental findings in Ref. [48]. Including the asymmetric CIFs leads to symmetry breaking of the heating profile along the current direction. Contrary to experiments on the gold chain, $E_{F}$ may in this case be tuned by gating. We predict the resulting hot spot to move from "downstream" to "upstream" with respect to the electron current when tuning from $E_{F}=1.4 \mathrm{eV}$ to $E_{F}=-1.0 \mathrm{eV}$ [Figs. 3(c) and 3(e) and 

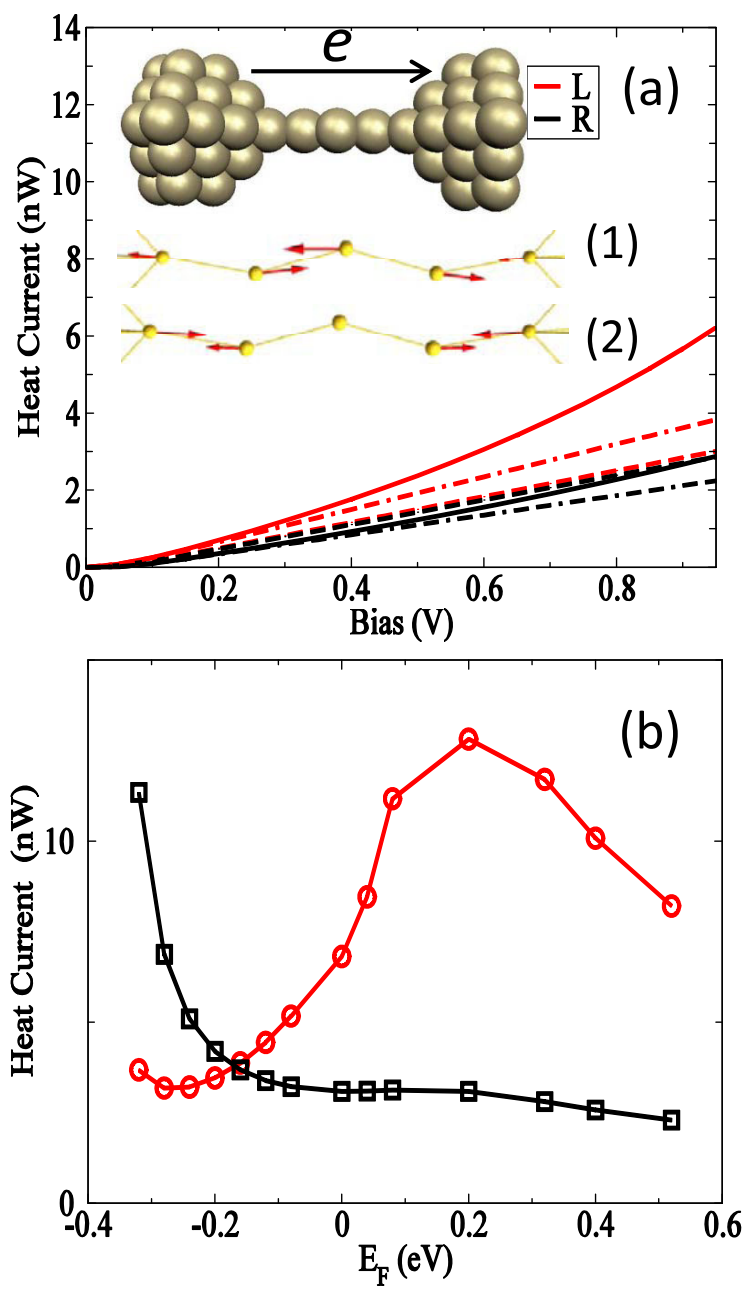

FIG. 2 (color online). (a) Bias dependence of the phonon heat current, going into the left and right phonon baths. Solid lines include the asymmetric CIFs $\left(\sim \operatorname{Im} \Lambda^{R L}\right)$, dashed lines do not, and the dash-dotted lines ignore the change of phonon spectral $\left(D^{r} / D^{a}\right)$ due to NC and BP forces. In the inset, we show the two vibrational modes that couple most strongly with the electrical current, with vibrational energy at (1)19 and (2) $18 \mathrm{meV}$. (b) Phonon heat current going into the left (red, circle) and right (black, square) baths at $V=1 \mathrm{~V}$, for different Fermi levels to illustrate the importance of the phase of the electron wave functions.

Fig. 3 in the Supplemental Material]. Thus, our calculation further suggests that which part of the edge bonds that breaks first may be controlled by gating.

The dependence of the hot spot on $E_{F}$ can be understood as follows (Sec. III of the Supplemental Material [32]). For a mirror-symmetric system with electron-hole symmetry, the asymmetric heating and heat flow is absent. When $E_{F}$ crosses the electron-hole symmetric point, the dominant current carriers contributing to inelastic transport change from electrons to holes, or vice versa. Thus, the hot spot moves from one side to the other. Interestingly enough, a similar effect in micrometer scale has been observed experimentally in graphene transistors $[50,51]$ and electrodes of molecular junctions [23]. Here, we show that it is equally important at atomic scale and related to the asymmetric CIFs.

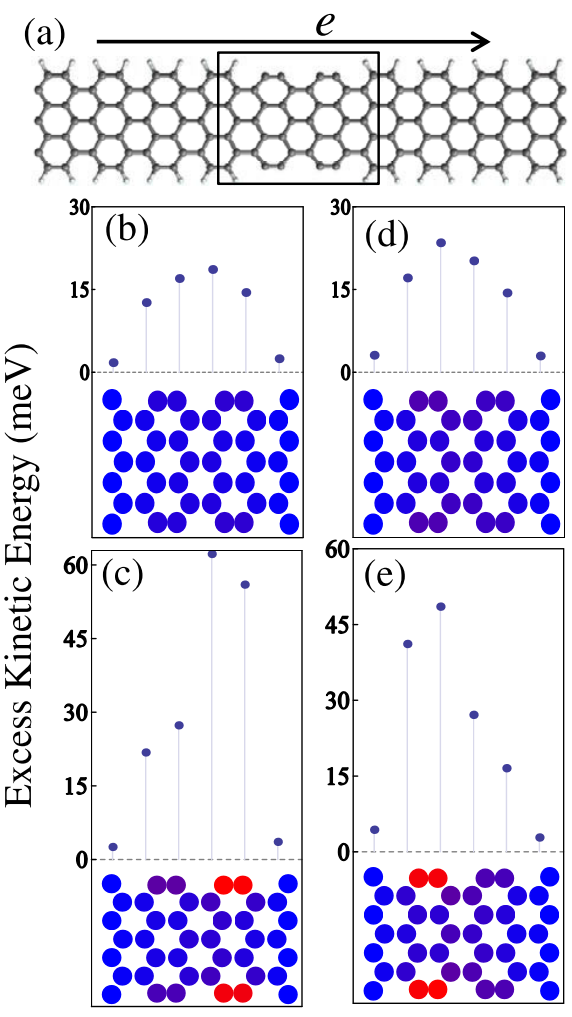

FIG. 3 (color online). (a) Structure of a partially passivated armchair graphene ribbon considered. The two sides of the ribbon are hydrogen passivated except in the device region, enclosed by the solid lines. [(b), (c)] The excess kinetic energy of each atom without and with the asymmetric CIFs, at $V=0.4 \mathrm{~V}$, $T=300 \mathrm{~K}, E_{F}=1.4 \mathrm{eV}$. The dots show the average over atoms belonging to each zigzag column. [(d), (e)] Same as with (b) and (c) with $E_{F}=-1.0 \mathrm{eV}$.

Scattering analysis.-The asymmetric heating and phonon heat flow at low bias can be qualitatively understood from the momentum transfer between electrons and phonons. To show this, we consider a simple one-dimensional model with a local e-ph interaction that involves the displacement of the $n$th and $n+1$ th atoms (junction) (Sec. IV of the Supplemental Material [32]),

$$
H_{\mathrm{e}-\mathrm{ph}}=\sum_{j \in\{n, n+1\}}-m \hat{u}_{j}\left(c_{j}^{\dagger} c_{j+1}-c_{j}^{\dagger} c_{j-1}+\text { H.c. }\right) \text {. }
$$

For $e V>0$, the important process is the inelastic electronic transition from the filled, left scattering states with momentum $k_{L}$ to the empty, right states with $k_{R}$. It is straightforward to show that the emission probability of a right-traveling phonon with momentum $q$ is different from that of a left-traveling mode $-q$, due to the difference in matrix elements for the processes,

$$
\Delta M_{L R}=\left|M_{L R}^{q}\right|^{2}-\left|M_{L R}^{-q}\right|^{2} \sim \sin (q) \sin \left(k_{L}-k_{R}\right) .
$$

Consequently, the left- and right-traveling steady-state phonon populations become different, resulting in asymmetric heat flow. 
In conclusion, we have presented a theory showing that CIFs in nanojunctions lead to asymmetric distributions and transport of the excess heat. We derived a Landauer-like formula for the excess heat transport. Employing firstprinciples calculations, we demonstrate that the size of the asymmetry can be crucial for current-induced processes at the atomic scale.

We thank T. N. Todorov, D. Dundas, and T. Markussen for discussions and the Danish Center for Scientific Computing (DCSC) for computer resources. This work is supported by the Lundbeck Foundation (R49-A5454), National Natural Science Foundation of China (Grants No. 11304107 and No. 61371015), and the Fundamental Research Funds for the Central Universities (HUST:2013TS032).

jitlu@hust.edu.cn

[1] We use phonons and vibrations interchangeably, although, strictly speaking, phonons are defined only in systems with translational invariance.

[2] D. Dundas, E. J. McEniry, and T. N. Todorov, Nat. Nanotechnol. 4, 99 (2009).

[3] J. T. Lü, M. Brandbyge, and P. Hedegård, Nano Lett. 10, 1657 (2010).

[4] N. Bode, S. V. Kusminskiy, R. Egger, and F. von Oppen, Phys. Rev. Lett. 107, 036804 (2011).

[5] T. N. Todorov, D. Dundas, A. T. Paxton, and A. P. Horsfield, Beilstein J. Nanotechnol. 2, 727 (2011).

[6] I. A. Pshenichnyuk and M.Č́ížek, Phys. Rev. B 83, 165446 (2011).

[7] R. Bustos-Marún, G. Refael, and F. von Oppen, Phys. Rev. Lett. 111, 060802 (2013).

[8] C. Schirm, M. Matt, F. Pauly, J. C. Cuevas, P. Nielaba, and E. Scheer, Nat. Nanotechnol. 8, 645 (2013).

[9] P. J. Wheeler, R. Chen, and D. Natelson, Phys. Rev. B 87, 155411 (2013).

[10] B. Cunningham, T. N. Todorov, and D. Dundas, Phys. Rev. B 90, 115430 (2014).

[11] N. J. Tao, Nat. Nanotechnol. 1, 173 (2006).

[12] M. Galperin, M. A. Ratner, and A. Nitzan, J. Phys. Condens. Matter 19, 103201 (2007).

[13] M. Galperin, M. A. Ratner, A. Nitzan, and A. Troisi, Science 319, 1056 (2008).

[14] Z. Huang, F. Chen, R. D’Agosta, P. A. Bennett, M. Di Ventra, and N. Tao, Nat. Nanotechnol. 2, 698 (2007).

[15] M. Tsutsui, M. Taniguchi, and T. Kawai, Nano Lett. 8, 3293 (2008).

[16] Y. Asai, Phys. Rev. B 78, 045434 (2008).

[17] R. H. M. Smit, Y. Noat, C. Untiedt, N. D. Lang, M. C. van Hemert, and J. M. van Ruitenbeek, Nature (London) 419, 906 (2002).

[18] W. Y. Wang, T. Lee, I. Kretzschmar, and M. A. Reed, Nano Lett. 4, 643 (2004).

[19] J. G. Kushmerick, J. Lazorcik, C. H. Patterson, R. Shashidhar, D. S. Seferos, and G. C. Bazan, Nano Lett. 4, 639 (2004).

[20] Z. Ioffe, T. Shamai, A. Ophir, G. Noy, I. Yutsis, K. Kfir, O. Cheshnovsky, and Y. Selzer, Nat. Nanotechnol. 3, 727 (2008).
[21] D. R. Ward, D. A. Corley, J. M. Tour, and D. Natelson, Nat. Nanotechnol. 6, 33 (2011).

[22] K. Kaasbjerg, T. Novotný, and A. Nitzan, Phys. Rev. B 88, 201405 (2013).

[23] W. Lee, K. Kim, W. Jeong, L. A. Zotti, F. Pauly, J. C. Cuevas, and P. Reddy, Nature (London) 498, 209 (2013).

[24] R. H. M. Smit, C. Untiedt, and J. M. van Ruitenbeek, Nanotechnology 15, S472 (2004).

[25] G. Schulze, K. J. Franke, A. Gagliardi, G. Romano, C. S. Lin, A. L. Rosa, T. A. Niehaus, T. Frauenheim, A. Di Carlo, A. Pecchia et al., Phys. Rev. Lett. 100, 136801 (2008).

[26] J.-T. Lü, M. Brandbyge, P. Hedegård, T. N. Todorov, and D. Dundas, Phys. Rev. B 85, 245444 (2012).

[27] J.-S. Wang, Phys. Rev. Lett. 99, 160601 (2007).

[28] R. P. Feynman and F. L. Vernon, Ann. Phys. (N.Y.) 24, 118 (1963).

[29] A. Caldeira and A. Leggett, Physica A (Amsterdam) 121A, 587 (1983).

[30] A. Schmid, J. Low Temp. Phys. 49, 609 (1982).

[31] Y. Oshima and Y. Kurui, Phys. Rev. B 87, 081404 (2013).

[32] See the Supplemental Material at http://link.aps.org/ supplemental/10.1103/PhysRevLett.114.096801 for details.

[33] J. Soler, E. Artacho, J. Gale, A. Garcia, J. Junquera, P. Ordejon, and D. Sanchez-Portal, J. Phys. Condens. Matter 14, 2745 (2002).

[34] M. Brandbyge, J. L. Mozos, P. Ordejon, J. Taylor, and K. Stokbro, Phys. Rev. B 65, 165401 (2002).

[35] T. Frederiksen, M. Paulsson, M. Brandbyge, and A.-P. Jauho, Phys. Rev. B 75, 205413 (2007).

[36] M. Engelund, M. Brandbyge, and A. P. Jauho, Phys. Rev. B 80, 045427 (2009).

[37] H. Yasuda and A. Sakai, Phys. Rev. B 56, 1069 (1997).

[38] H. Ohnishi, Y. Kondo, and K. Takayanagi, Nature (London) 395, 780 (1998).

[39] A. I. Yanson, G. R. Bollinger, H. E. van den Brom, N. Agraït, and J. M. van Ruitenbeek, Nature (London) 395, 783 (1998)

[40] J. T. Lü, P. Hedegård, and M. Brandbyge, Phys. Rev. Lett. 107, 046801 (2011).

[41] Y. Dubi and M. Di Ventra, Phys. Rev. B 79, 115415 (2009).

[42] Y. Dubi and M. Di Ventra, Phys. Rev. E 79, 042101 (2009).

[43] P. A. Jacquet, J. Stat. Phys. 134, 709 (2009).

[44] P. A. Jacquet and C.-A. Pillet, Phys. Rev. B 85, 125120 (2012).

[45] J. P. Bergfield, S. M. Story, R. C. Stafford, and C. A. Stafford, ACS Nano 7, 4429 (2013).

[46] Another way of quantifying the heating is to use the local temperature defined in some way. We tried to use the method in Refs. [40-44]. The result is shown in Fig. 3 of the Supplemental Material [32]. The overall heating profile agrees with Fig. 1.

[47] T. Frederiksen, M. Brandbyge, N. Lorente, and A.-P. Jauho, Phys. Rev. Lett. 93, 256601 (2004).

[48] X. Jia, M. Hofmann, V. Meunier, B. G. Sumpter, J. CamposDelgado, J. M. Romo-Herrera, H. Son, Y.-P. Hsieh, A. Reina, J. Kong et al., Science 323, 1701 (2009).

[49] M. Engelund, J. A. Fürst, A. P. Jauho, and M. Brandbyge, Phys. Rev. Lett. 104, 036807 (2010).

[50] M. Freitag, H.-Y. Chiu, M. Steiner, V. Perebeinos, and P. Avouris, Nat. Nanotechnol. 5, 497 (2010).

[51] M.-H. Bae, Z.-Y. Ong, D. Estrada, and E. Pop, Nano Lett. 10, 4787 (2010). 